\title{
Depression and heart failure: an intricate relationship
}

\author{
Gaetano Aloisi ${ }^{1}$, Alberto Zucchelli ${ }^{1}$, Bruno Aloisi ${ }^{2}$, Giuseppe Romanelli ${ }^{3}$, Alessandra Marengoni ${ }^{3}$ \\ ${ }^{1}$ School of Geriatrics, University of Brescia; ${ }^{2}$ Cardiology Specialist, Catania; ${ }^{3}$ Department of Clinical and \\ Experimental Sciences, University of Brescia, Italy
}

\begin{abstract}
In patients with heart failure (HF), depression is common and associated with adverse outcomes such as reduced adherence to treatment, poor quality of life, increased hospitalizations and elevated mortality. Despite these adverse impacts, depression remain underdiagnosed in HF patients. We performed a target review of the literature to identify the association between HF and depression, to examine the mechanisms that link these two conditions and to identify instruments for an accurate diagnosis and treatment of depression in HF patients. Depression is associated with the development and progression of HF, including increased rates of mortality, mediated by behavioral and pathophysiological mechanisms. The over-
\end{abstract}

Correspondence: Gaetano Aloisi, School of Geriatrics, University of Brescia, Piazza del Mercato 15, 25121 Brescia, Italy.

E-mail: gaetano.aloisi@gmail.com

Contributions: GA, study concept and design; GA, AZ, manuscript drafting; GA, AZ, BA, GR, AM, critical revision of the manuscript and substantive intellectual contribution. All authors made a substantive intellectual contribution, and read and approved the final version of the manuscript.

Conflict of Interest: All authors declare that no organizations have attempted to influence the study or the writing of the manuscript. They have had no financial relationships in the previous three years with any organizations that might have an interest in influencing the submitted work and no other relationships or activities that could appear to have influenced the submitted work.

Transparency Statement: The lead author (GA) affirms that the manuscript is an honest, accurate, and transparent account of the study being reported; that no important aspects of the study have been omitted; and that any discrepancies from the study as originally planned have been explained.

Key words: Heart failure; depression; screening; antidepressants.

Received for publication: 3 January 2019.

Accepted for publication: 5 August 2019.

${ }^{\circ}$ Copyright: the Author(s), 2019

Licensee PAGEPress, Italy

Monaldi Archives for Chest Disease 2019; 89:1029

doi: 10.4081/monaldi.2019.1029

This article is distributed under the terms of the Creative Commons Attribution Noncommercial License (by-nc 4.0) which permits any noncommercial use, distribution, and reproduction in any medium, provided the original author(s) and source are credited. lap of symptoms between depression and HF often makes the diagnosis of depression difficult and late. Currently, specific guidelines for depression screening in HF patients are lacking, partly because evidences showing that depression screening improves cardiac outcomes are insufficient. European guidelines suggest the early use of instruments such as the Beck Depression Inventory (BDI) and the Geriatric Depression Scale (GDS), both characterized by accuracy and administration simplicity. There is limited evidence of pharmacological treatment and psychotherapy efficacy in patients with HF. However, cognitive-behavioral therapy has been shown to improve outcomes HF patients, and selective serotonin reuptake inhibitors appear safe in this cohort.

\section{Introduction}

Heart failure (HF) is a worldwide prevalent disease. An estimated 5.7 million Americans are affected by heart failure [1], and there are at least 15 million patients with heart failure among the 51 countries represented by the European Society of Cardiology (ESC) [2]. Furthermore, HF is a major cause of death and disability [3].

Depression is common in $\mathrm{HF}$, with a prevalence range of 11$51 \%$ [4], and it is one of the main contributors to poor outcome in HF patients [5,6]. Depression has been independently associated with poor quality of life, functional limitations, and an increased risk of morbidity and mortality in the HF population [7-9].

\section{Depression and HF}

The relationship between depression and HF is complex being due to both behavioral and pathophysiological factors as shown in Figure 1. Symptoms of depression such as fatigue, lack of motivation and social isolation affect patients' adherence to HF treatment. Furthermore, depression and HF have several pathophysiological mechanisms in common, such as chronic inflammation, oxidative stress, and dysregulation of the hypothalamic-pituitaryadrenal axis [10]. Furthermore, this association is bidirectional. $\mathrm{HF}$, especially in NYHA III-IV classes, often causes poor quality of life due to troublesome physical symptoms (e.g. dyspnea, fatigue, and sleeping disorders), unplanned hospitalizations and poor functional status. Finally, studies have observed the role of the hippocampus in mediating several neuropsychological functions, as well as in the pathogenesis of depression and cognitive impairment. Hippocampus, being a brain highly vascular structure, is vulnerable to the hypoxic injury caused by the acute exacerbations of heart failure [11]. Therefore, a significant proportion of HF patients are expected to develop depression during the disease course. Additionally, a significant number of those with 
depression are at greater risk of worse HF outcomes (Figure 2).

\section{Importance of screening for depression in HF patients}

The overlap of symptoms (e.g. fatigue, anorexia, weight changes, sleep loss) between depression and HF [12] often makes depression diagnosis difficult and late. Currently, specific guidelines for depression screening in HF patients are lacking, also because there is insufficient evidence showing that depression screening improves cardiac outcomes [13]. The American Heart Association (AHA) has issued a recommendation for depression screening in patients with coronary heart disease (CHD), the most common cause of HF [14]. They recommend to routinely screen for depression patients with CHD in various settings (i.e., hospitals, clinics, and cardiac rehabilitations centers), using different tools (Patient Health Questionnaire 2 and 9 [15], PHQ-2 [16], and PHQ-9). On the other hand, European guidelines for HF suggest the early use of questionnaires such as the Beck Depression Index (BDI) [17] and the Geriatric Depression Scale (GDS) [18]. During the last Seminary of the Italian Geriatric Cardiology Society (SICGe), held in Rome in June 2018, after a panel discussion, the faculty agreed that the 15-items GDS could be the first choice for elderly with HF, considering its accuracy and administration simplicity. Furthermore, the GDS does not provide for questions related to the patient's physical state but only specifically to the mood. Elderly clinical conditions could influence the results of other scales that include questions on somatic symptoms related to depression.
Salient points regarding screening for depression in $\mathrm{HF}$ patients have been summarized in Table 1 .

\section{Treatment of depression in $\mathbf{H F}$}

Several studies have shown the outcome of different treatment options for depression, e.g. pharmacotherapy, psychotherapy (including cognitive behavioral therapy) and exercise training, in HF patients. However, prospective randomized controlled trials are still lacking.

\section{Antidepressants}

Several drugs can be used to treat depression in HF patients; the choice of the pharmacological class should take into account both comorbidities and potential drug-drug (DDIs) and drug-disease interactions. First-generation antidepressants, including monoamine oxidase inhibitors (MAOIs) and tricyclic antidepressants (TCAs), have been associated with a significant risk of potentially harmful pharmacodynamic DDIs causing hypertensive crisis and serotonin syndrome when prescribed with MAOIs [19]. Furthermore, patients prescribed with TCAs [20] are at increased risk of QT interval prolongation and arrhythmias, such as "torsades de pointes".

Second-generation antidepressants are less likely to cause pharmacodynamic DDIs, given a more selective mechanism of action and receptor profile. Clinically significant pharmacokinetic DDIs include an enhanced anticoagulant effect and an increased risk of bleeding when fluoxetine or fluvoxamine (both potent inhibitors of cytochrome $\mathrm{P} 2 \mathrm{C} 9$ ) are combined with warfarin [21].

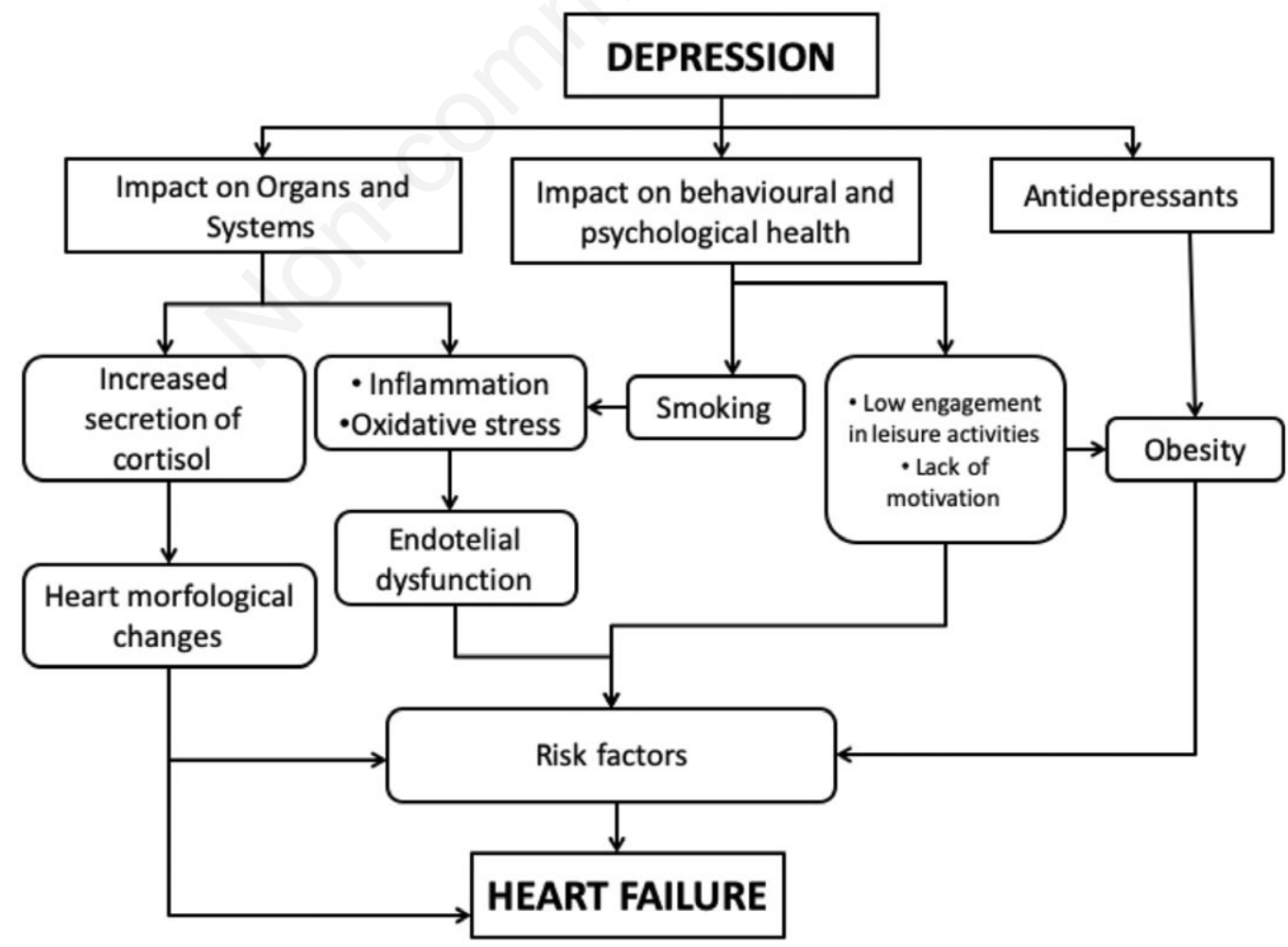

Figure 1. Intricate pathophysiological relationship between depression and HF. 
Severe bradycardia and heart block have been reported when metoprolol and propranolol (both substrates of cytochrome P2D6) were prescribed in combination with fluoxetine [22].

Based on the safety data, a SSRI should be used as the first-line therapy [23], but the efficacy of antidepressant therapy on the outcomes of patients with HF is controversial. The SADHART-CHF (Sertraline Against Depression and Heart Disease in Chronic Heart Failure) trial selected 469 patients with HF and major depression, who were administered sertraline or placebo for 12 weeks. HF patients who achieved remission of depressive symptoms fared better on the assessment of physical function and quality of life as well. Depression remission was in fact significantly associated with higher improvements in Kansas City Cardiomyopathy Questionnaire subscale scores $(\mathrm{p}<0.001)$ and in the 6-minute walk test performances $(p=0.03)$ [24]. Paradoxically, treating depression with antidepressants versus placebo does not seem to be a guaran- tee for improved overall outcome. The proportion whose composite cardiovascular score worsened, improved, or was unchanged was $29.9 \%, 40.6 \%$, and $29.5 \%$ in the sertraline group and $31.1 \%$, $43.8 \%$, and $25.1 \%$ in the placebo group $(p=0.78)$ [25]. On the other hand, results from the MOOD-HF (The Morbidity, Mortality and Mood in Depression Heart Failure patients) study showed that 18 months of treatment with escitalopram $(n=185)$ versus placebo $(n=187)$ failed to significantly reduce the hospitalization and allcause mortality in patients with $\mathrm{HF}$ and reduced ejection fraction (LVEF $<45 \%$ ). The endpoint occurred equally in 116 patients $(63 \%)$ in the escitalopram group and in 119 patients $(64 \%)$ in the placebo group $(p=0.92)$ [26]. A recent review showed that antidepressants are not associated with increased mortality rate, as reported by previous papers. However, evidences of antidepressants' effects on depression symptoms' and cardiac outcomes' is still lacking [27].

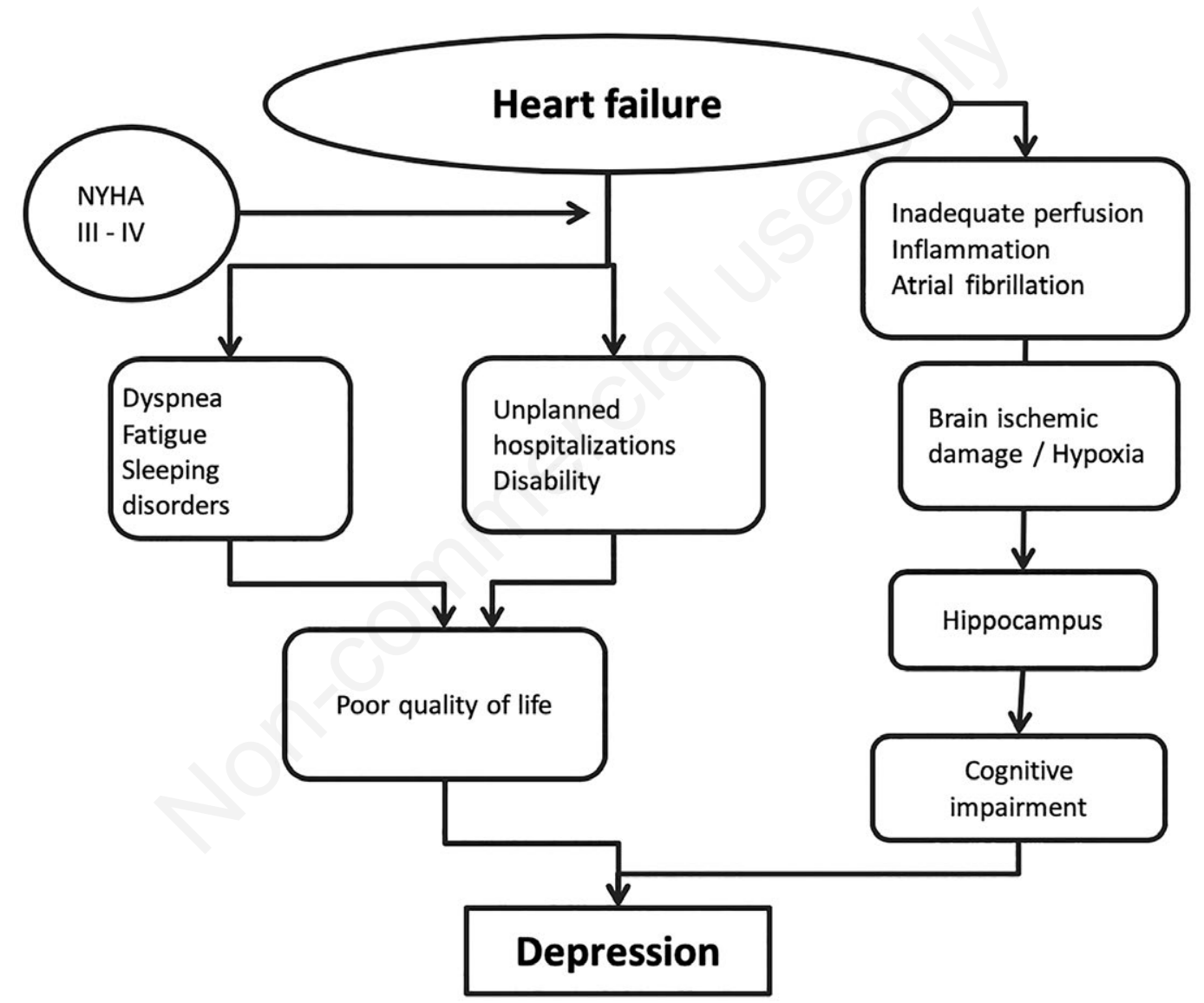

Figure 2. Heart failure leading to depression.

Table 1. Screening for depression in heart failure.

- Early detection of depression is warranted

- Diagnosis of depression is challenging because of symptoms' overlap

- Several tools are available for depression screening (PHQ-2 and PHQ-9, BDI, GDS)

- AHA recommends screening for depression in CHD patients

- ESC suggests screening for depression in HF patients with BDI or GDS questionnaires

- Specific guidelines for depression screening in HF patients are lacking

HF, heart failure; PHQ, patient health questionnaire; BDI, Beck depression index; GDS, geriatric depression scale; AHA, American Heart Association; CHD, coronary heart disease; ESC, European Society of Cardiology. 
Table 2. Effects of treatment interventions on depression in heart failure.

Antidepressants in HF

- Treating depression with antidepressant may not always improve HF outcomes

- SSRIs are the safest antidepressants in HF

- Several potential drug-drug interactions between antidepressants and HF medications are known

- MOOD-HF and SADAHRT-CHF trials showed controversial results

Cognitive behavioral therapy

- Improvement was observed in physical function, quality of life and cardiac event-free survival

Exercise therapy

- Aerobic mode was found beneficial for depression in HF patients

- Favorable effects on hypercortisolism, inflammation, endothelial dysfunction, hypercoagulability

HF, heart failure; MOOD-HF, The Morbidity, Mortality and Mood in Depression Heart Failure patients; SADAHRT-CHF, Sertraline Against Depression and Heart Disease in Chronic Heart Failure

Studies about the efficacy of vortioxetine to reduce symptoms and improve outcomes in depressed patients with HF are still lacking, but safety data show a cardiovascular safety profile similar to placebo. In a recent study, Vortioxetine had no effect to heart rate and blood pressure and showed no relevant effects on ECG parameters, including the QTc interval [28].

\section{Psychotherapy}

Few studies have evaluated the role of cognitive behavioral therapy (CBT) for depression treatment in HF [29,30] showing some improvement in physical function, quality of life and 3month cardiac event-free survival. Its effect on HF symptoms is controversial as not all studies reported improvement in this measure [31]. In an interesting meta-analysis by Jeyanantham et al. including 6 papers, CBT appears to be more effective than usual care at improving quality of life in heart failure patients. Anyhow, the effect was not sustained 3 months later. There were no observable differences between CBT and usual care for hospitalizations or mortality [32].

\section{Exercise}

Compared with guideline-based usual care, exercise training resulted in a modest reduction in depressive symptoms in patients with HF [33]. It increases the release of neurotransmitters (serotonin, dopamine, and norepinephrine) which improve depressive symptoms. The presence of depression has been linked to hypercortisolism, sympathetic over-activity, inflammation, endothelial dysfunction, and hypercoagulability, all of which worsen symptoms of HF. Exercise has been found to have a favorable effect on all these factors, and it also improves survival in patients with HF [34].

Salient points regarding treatment of depression in HF patients have been summarized in Table 2 .

\section{Conclusions}

Heart failure is one of the major worldwide chronic health problems, affecting survival and quality of life in a large number of individuals. Depression has been found to be highly prevalent in this population, and it further worsens patients' prognosis. Early detection using screening tools is critical, suggesting the need of guidelines for depression screening in HF patients. Despite different therapeutic options have been proposed so far, prospective studies are needed to evaluate the best pharmacological approach for patients with co-occurring depression and HF. Similarly, CBT may be specifically designed for HF patients with depression given their focus on negative thoughts and the emotional burden of having HF. Exercise training has been shown to reduce depressive symptoms in patients with HF and reduced ejection fraction, but further prospective studies are needed to prove the effectiveness of this therapeutic approach across different subgroups of patients.

\section{References}

1. Lloyd-Jones D, Adams R, Carnethon M, et al. Heart Disease and Stroke Statistics--2009 Update: A Report From the American Heart Association Statistics Committee and Stroke Statistics Subcommittee. Circulation 2009;119:e21-e181.

2. Dickstein K, Cohen-Solal A, Filippatos G, et al. ESC Guidelines for the diagnosis and treatment of acute and chronic heart failure 2008: the Task Force for the Diagnosis and Treatment of Acute and Chronic Heart Failure 2008 of the European Society of Cardiology. Developed in collaboration with the Heart Failure Association of the ESC (HFA) and endorsed by the European Society of Intensive Care Medicine (ESICM). Eur Heart J 2008;29:2388-442.

3. Vader JM, LaRue SJ, Stevens SR, et al. Timing and causes of readmission after acute heart failure hospitalization - insights from the heart failure network trials. J Card Fail 2016;22: 875-883.

4. Rutledge T, Reis VA, Linke SE, et al. Depression in heart failure a meta-analytic review of prevalence, intervention effects, and associations with clinical outcomes. J Am Coll Cardiol 2006;48:1527-37.

5. Levy WC, Mozaffarian D, Linker DT, et al. The Seattle Heart Failure Model: prediction of survival in heart failure. Circulation 2006;113:1424-33.

6. Levy WC, Linker DT. Prediction of mortality in patients with heart failure and systolic dysfunction. Curr Cardiol Rep 2008;10:198-205.

7. Muller-Tasch T, Peters-Klimm F, Schellberg D, et al. Depression is a major determinant of quality of life in patients with chronic systolic heart failure in general practice. J Card Fail 2007;13:818-24.

8. O'Connor CM, Jiang W, Kuchibhatla M, et al. Antidepressant use, depression, and survival in patients with heart failure. Arch Intern Med 2008;168:2232-7.

9. Gottlieb SS, Kop WJ, Ellis SJ, et al. Relation of depression to severity of illness in heart failure (from Heart Failure and a Controlled Trial Investigating Outcomes of Exercise Training [HFACTION]). Am J Cardiol 2009;103:1285-9.

10. Ghosh RK, Ball S, Prasad V, Gupta A. Depression in heart fail- 
ure: Intricate relationship, pathophysiology and most updated evidence of interventions from recent clinical studies. Intern J Cardiol 2016;224:170-7.

11. Woo MA, Ogren JA, Abouzeid CM, et al. Regional hippocampal damage in heart failure. Eur J Heart Fail 2015;17:494-500.

12. Cully JA, Jimenez DE, Ledoux TA, et al. Recognition and treatment of depression and anxiety symptoms in heart failure. Prim Care Companion J Clin Psychiatry 2009;11:103-9.

13. Thombs BD, Roseman M, Coyne JC, et al. Does evidence support the American Heart Association's recommendation to screen patients for depression in cardiovascular care? An updated systematic review. PLoS One 2013;8:e52654.

14. Lichtman JH, Bigger Jr. JT, Blumenthal JA, et al. Depression and coronary heart disease: recommendations for screening, referral, and treatment: a science advisory from the American Heart Association Prevention Committee of the Council on Cardiovascular Nursing, Council on Clinical Cardiology, Council on Epidemiology and Prevention, and Interdisciplinary Council on Quality of Careand Outcomes Research: endorsed by the American Psychiatric Association. Circulation 2008;118:1768-75.

15. Spitzer RL, Kroenke K, Williams JB. Validation and utility of a self-report version of PRIME-MD: the PHQ primary care study. Primary Care Evaluation of Mental Disorders. Patient Health Questionnaire. JAMA 1999;282:1737-44.

16. Kroenke K, Spitzer RL, Williams JB The Patient Health Questionnaire-2: validity of a two-item depression screener. Med Care 2003;41:1284-92.

17. Richter P, Werner J, Heerlein A, Kraus A, et al. On the validity of the Beck Depression Inventory. A review. Psychopathology 1998;31:160-8

18. Ponikowski P, Voors AA, Anker SD, et al. 2016 ESC Guidelines for the diagnosis and treatment of acute and chronic heart failure: the Task Force for the diagnosis and treatment of acute and chronic heart failure of the European Society of Cardiology (ESC). Developed with the special contribution of the Heart Failure Association (HFA) of the ESC. Eur J Heart Fail 2016;18:891-975.

19. Grossman E, Messerli FH. High blood pressure: a side effect of drugs, poisons, and foods. Arch Intern Med 1995;155:450-60.

20. Glassman AH. Cardiovascular effects of antidepressant drugs: updated. Int J Clin Psychopharmacol 1998;13:S25-S30.

21. Dent LA, Orrock MW. Warfarin-fluoxetine and diazepam-fluoxetine interaction. Pharmacotherapy 1997;17:170-2.

22. Drake WM, Gordon GD. Heart block in a patient on propranolol and fluoxetine. Lancet 1994;343:425-6.

23. Watson K, Summers KM. Depression in patients with heart fail- ure: clinical implications and management. Pharmacotherapy 2009;29:49-63.

24. Xiong GL, Fiuzat M, Kuchibhatla M, et al. Health status and depression remission in patients with chronic heart failure: patient-reported outcomes from the SADHARTCHF trial. Circ Heart Fail 2012;5:688-92.

25. O'Connor CM, Jiang W, Kuchibhatla M, et al. Safety and efficacy of sertraline for depression in patients with heart failure: results of the SADHART-CHF (sertraline against depression and heart disease in chronic heart failure) trial. J Am Coll Cardiol 2010;56:692-9.

26. Angermann CE, Gelbrich G, Störk S, et al. Effect of escitalopramon all-cause mortality and hospitalization in patients with heart failure and depression: the MOOD-HF randomized clinical trial. JAMA 2016; 315:2683-93.

27. Rajeswaran T, Plymen CM, Doherty AM. The effect of antidepressant medications in the management of heart failure on outcomes: mortality, cardiovascular function and depression a systematic review. Int J Psychiatry Clin Pract 2018;22:164-9.

28. Baldwin DS, Chrones L, Florea I, et al. The safety and tolerability of vortioxetine: Analysis of data from randomized trials and open-label extension study. J Psychopharmacol 2016;3:242-52.

29. Tully PJ, Selkow T, Bengel J, et al. A dynamic view of comorbid depression and generalized anxiety disorder symptom change in chronic heart failure: the discrete effects of cognitive behavioral therapy, exercise, and psychotropic medication. Disabil Rehabil 2015;37:585-92.

30. Freedland KE, Carney RM, Rich MW, et al. Cognitive behavior therapy for depression and self-care in heart failure patients: a randomized clinical trial. JAMA Intern Med 2015; 175:1773-82.

31. Dekker RL, Moser DK, Peden AR, et al. Cognitive therapy improves three-month outcomes in hospitalized patients with heart failure. J Card Fail 2012;18:10-20.

32. Jeyanantham K, Kotecha D, Thanki D, et al. Effects of cognitive behavioural therapy for depression in heart failure patients: a systematic review and meta-analysis. Heart Fail Rev 2017;22:731-41.

33. Blumenthal JA, Babyak MA, O'Connor C, et al. Effects of exercise training on depressive symptoms in patients with chronic heart failure: the HF-ACTION randomized trial. JAMA 2012;308:465-74.

34. Milani RV, Lavie CJ, Mehra MR, et al. Impact of exercise training and depression on survival in heart failure due to coronary heart disease. Am J Cardiol 2011;107:64-8. 